\title{
PROSPECTS OF THE GERMAN MULTIBODY SYSTEM RESEARCH PROJECT ON VEHICLE DYNAMICS SIMULATION
}

\author{
Werner Schiehlen \\ Institute B of Mechanics, University of Stuttgart, Stuttgart, Germany
}

\begin{abstract}
SUMMARY
The German Research Council (DFG) decided 1987 to establish a nationwide research project devoted to dynamics of multibody systems. In this project 14 universities and research centers are cooperating with the goal to develop a general purpose multibody system software package. This concept provides the opportunity to use a modular structure of the software, i.e. different multibody formalisms may be combined with different aimulation programes via standardized interfaces. For the DFG project the database RSYST was chosen using standard FORTRAN 77 and an object oriented multibody system datamodel was defined. According to the modular concept the requirements of vehicle system dynamics as tire models or railway wheel-rail models, respectively, are easily met. The Iltis benchmark problem is used to demonstrate some features of the object oriented datamodel.
\end{abstract}

\section{INTRODUCTION}

Mechanical systems like rail or road vehicles can be modeled properly as multibody systems. The complexity of the dynamical equations called for the development of computer-aided formalisms a quarter of a century ago. The state-of-the-art was presented at a series of IUTAM/IAVSD symposia, documented in the corresponding proceedings, see, e.g., Magnus [1], slibar und springer [2], Haug [3], Kortüm and Schiehlen [4], Bianchi and Schiehlen [5], Kortüm and Sharp [6]. In addition, a number of commercially available computer codes was developed, a summary of which is given in the Multibody System Handbook [7] and the forthcoming Report on Herbertov Multibody Workshop [8]. The computer codes available show different capabilities: some of them generate only the equations of motion in numerical or symbolical form, respectively, some of them provide numerical integration or simulation codes, too. Moreover, there are also extensive software systems on the market which offer additionally graphical data input, animation of body motions and automated signal data analysis. There is no doubt that the professional user, particularly in the automotive industry, prefer the most complete software system for dynamical multibody system analysis. 
The development of large software aystems in the field of multibody dynamics means interdisciplinary research of several groups of acientists. Therefore, the German Research Council (DFG) decided in 1987 to establish such a nationwide research project.

The project includes

- research on the fundamentals of the method of multibody systems,

- concepts for new formalisms of dynamical analysis,

- development of efficient numerical algorithms and

- realization of a powerful software package of multibody systems.

The goals require an interdisciplinary cooperation between mathematicians, computer scientists, mechanicists and control engineers. The following research institutions are participating in the project (under the responsibility of leading scientists):

- Technical University of Aachen (Prof. G. Sedlacek)

- University of Augaburg (Prof. H. G. Bock)

- Technical University of Darmstadt (Prof. P. Hagedorn)

- Univergity of Duisburg (Prof. M. Hiller)

- University of German Army, Hamburg (Prof. L. Gaul, Dr. B. Zastrau)

- University of Hannover (Dr. B. O. Dirr, Prof. K. Popp)

- University of Karlsruhe (Prof. J. Wittenburg, Dr. U. Wolz)

- Technical University of Munich (Prof. H. Bremer, Prof. F. Pfeiffer)

- MAN Technology Coorporation, Munich (Dr. P. Meinke)

- German Research Institute for Aerospace (DLR), Oberpfaffenhofen (Dr. W. Kortüm, Dr. R. Schwertassek)

- University of Paderborn (Prof J. Lückel)

- University of Stuttgart (Prof. E. Kreuzer, Hamburg and Prof. W. Schiehlen)

- Computing Center of the Univeraity of Stuttgart (Prof. R. Rühle)

- Univerbity of Wuppertal (Prof. P. C. Müller)

In 1989 it was decided to use a database concept following software engineering principles as shown in Fig. 1. This concept provides the opportunity to use a modular etructure of the software, 1.e. different multibody formalisms may be combined with different simulation programmes via standardized interfaces. For the DFG project the database RSYST was chosen using standard FORTRAN 77 or ADA, respectively.

The following modulg of the software package are under development:

Graphical Preprocessor(MBKGAM, MBW...)

Generation of Dynamical Equations (MBNNEF, MBRGEN)

Modeling of Elastic Elementg (MBE..., MBSFLX)

Finite Element Applications (MBH...)

Nonlinear Elastic Beams (MBG...)

Modeling of Viscoelastic Elements (MBC..., MBD...)

Numerical simulation (MBP...., MBF...)

Advanced Simulation Techniques (MBU...)

Graphical Animation (MBKGAM, MBW...) 


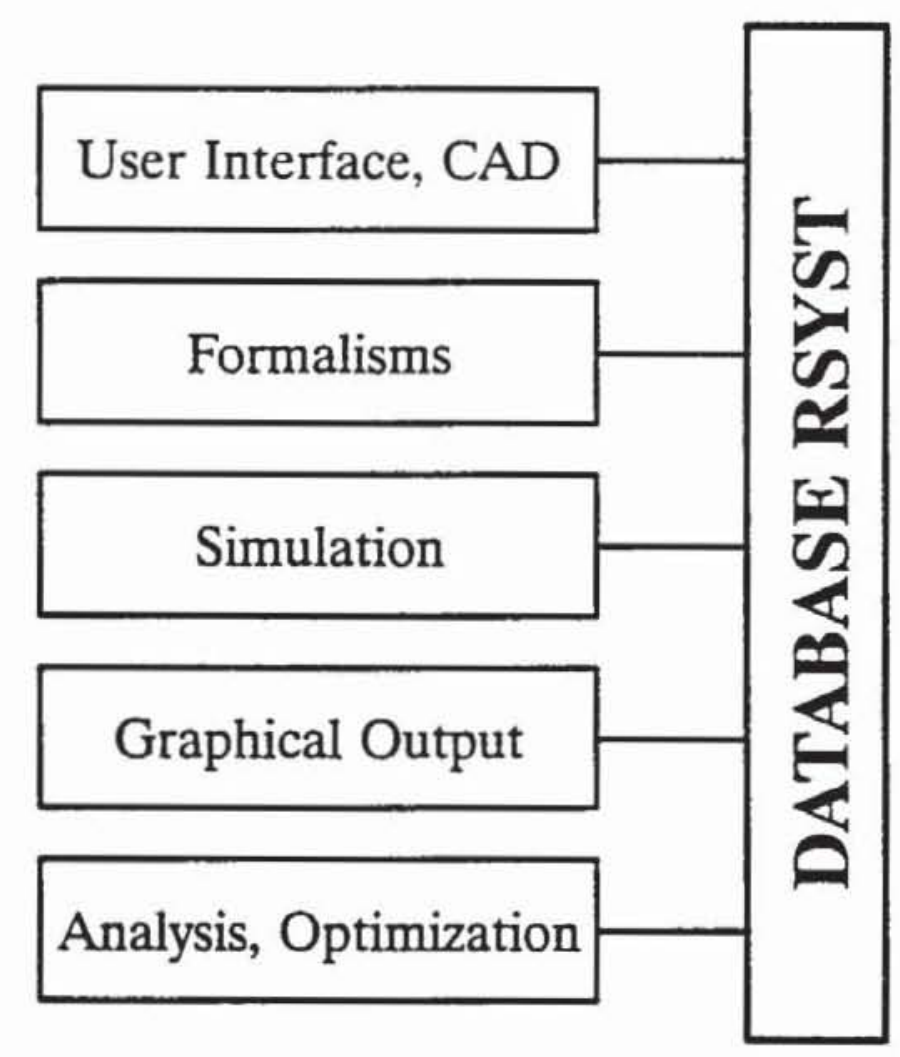

Fig. 1. Software Engineering Concept of the Multibody System Research Project

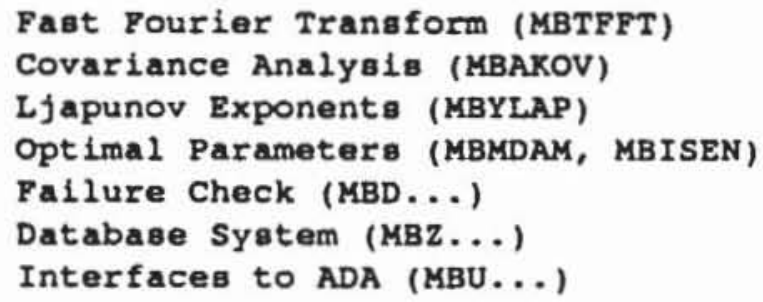

A recent survey on the project is presented by Meinke [9], the corporation between the participating institutions is shown in Fig. 2 .

The DFG project is open for special moduls required in vehicle system dynamics as tire models or railway wheel-rail models, respectively, according to the modular concept. On the other hand, the database background of the project will not allow any realtime simulations, sometimes necessary in vehicle dynamics, too. However, the efficiency of formalisms or simulation software, respectively, may be more easily teated in the project software environment before applied in special realtime codes. This is a major advantage of the standardized interfaces. 


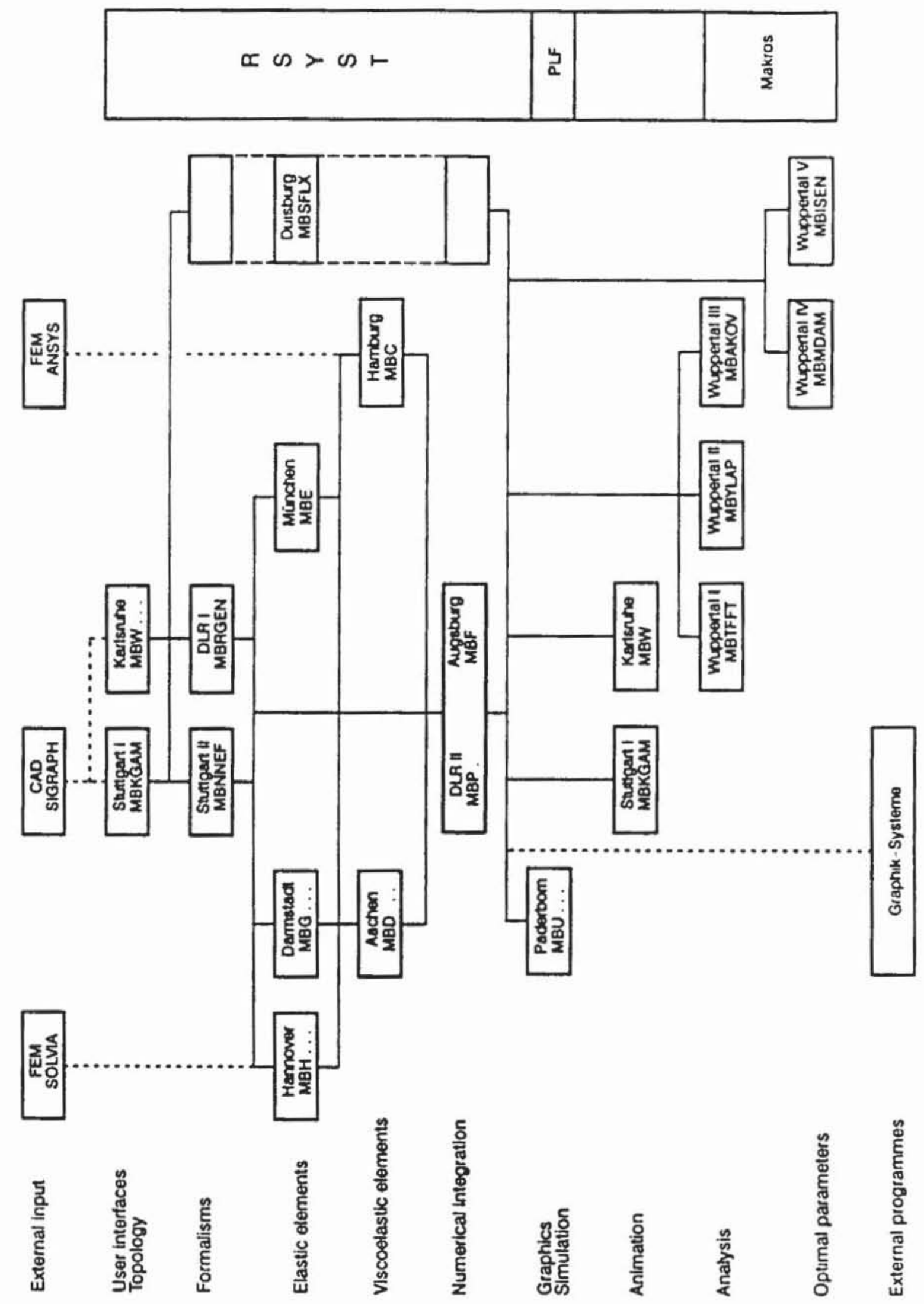

Fig. 2. Coorporation within the Multibody systems Research Project 


\section{THE DATABASE RSYST}

The scientific-engineering database system RSYST is considered to be a software tool. It aupports the

- development of user programmes,

- development of program packages,

- execution of programmea,

- handling of large data sets,

- analysis of data.

One of the main applications of RSYST is the compilation of data and user programmes. RSYST is written in FORTRAN 77 and, therefore, it has an excellent portability to all kinds of computers. The fundamental elements of RSYST are the following:

- execution control,

- information system,

- dialogue system,

- output handling,

- dynamic storage handling,

- method and model base,

- database.

The RSYST system has been developed by Rühle and his staff at the Computing Center of the University of Stuttgart. A detailed description is given by Lang [10], Loebich [11] and Rühle [12].

The possibilities for the compilation of user programmes with RSYST is shown in Fig. 3. Most effective is the complete integration of a user modul in the RSYST environment regarding all the interface definitions. In other савев, the simple integration or a weak compilation may be appropriate.

Most important for the multibody systems project are the RSYST database and the handling of data objects. All the data in RSYST are considered as objects of a database. Such data objects are e.g. vectors, matrices, sets of parameters, texts or formally defined objects. The data objects are stored in the RSYST database subject to a very efficient handling, they are identified by special names.

Each data object in the RSYST database is characterized by a data description, identifying to the data type. The data description permits a correlation between data objects and possible operations.

RSYST offers the following operations on data objects which are completely internal executed:

- object generating,

- object changing,

- object deleting,

- object listing,

- objects relating to each other,

- objects storing and reading,

- handling of components of objects. 
I

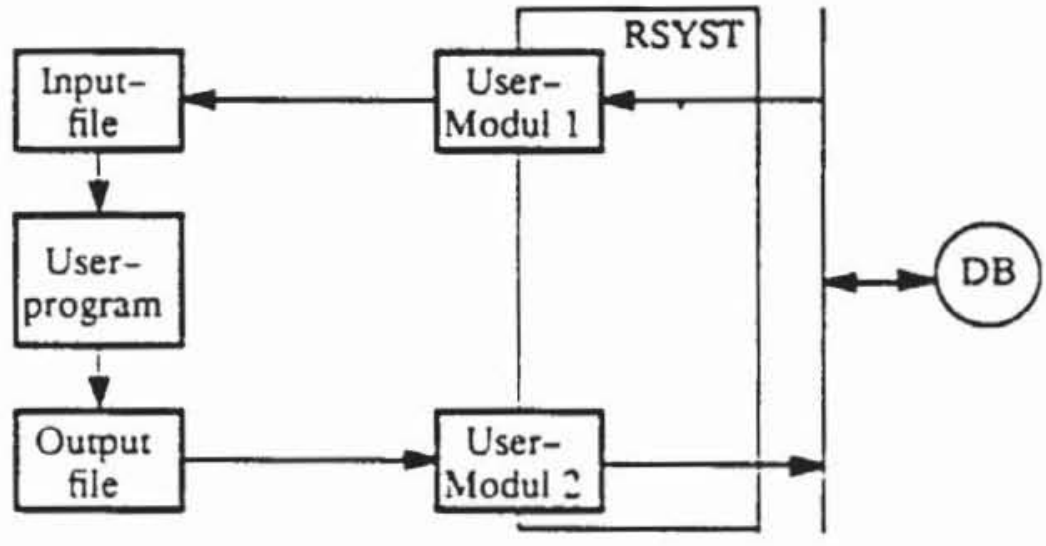

IIa

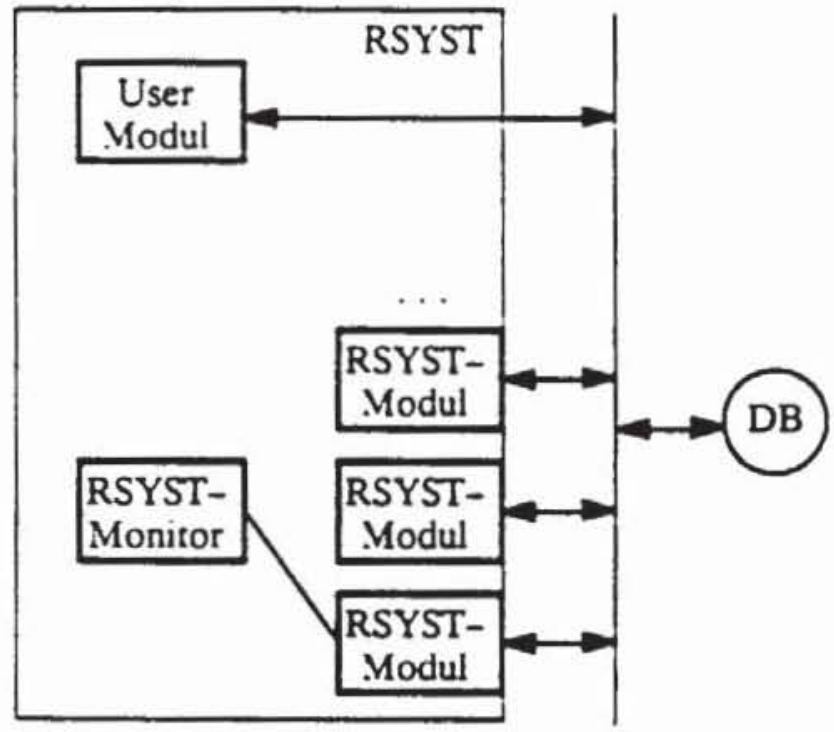

IIb

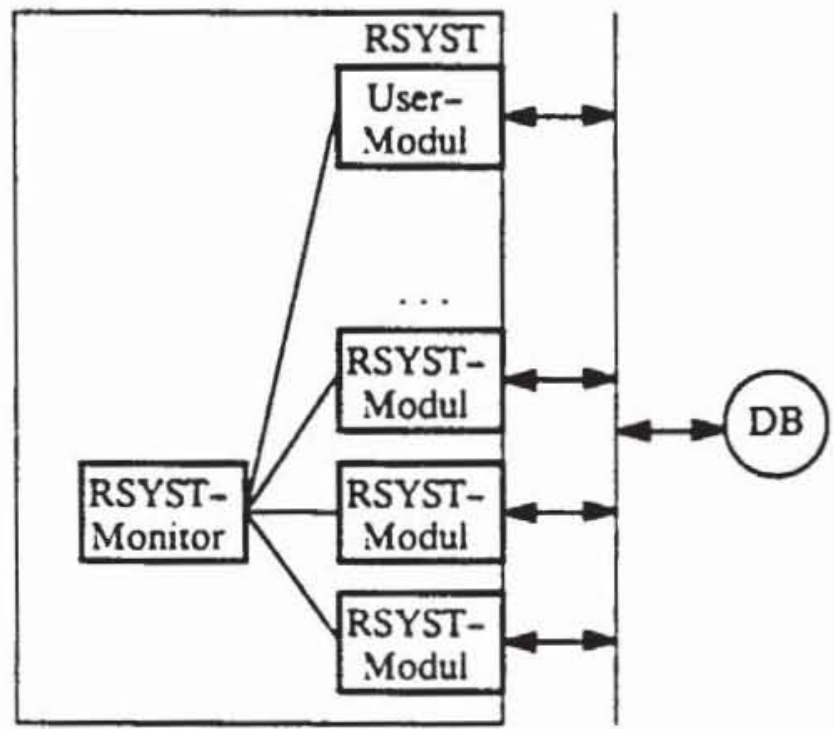

Fig. 3. Possibilty of programe compilation with RSYST

I) weak compilation

IIa) simple integration (stand-alone-modul)

IIb) complete integration (subsystem) 
The objects have to be interpreted for the identification of their information. A set of objects with same rules of interpretation are called a class specified by a name. For example, the four components of the class "frame", are shown in Fig. 4.

\begin{tabular}{|l|c|c|c|c|c|l|}
\hline class: & frame \\
\hline name & type & $\min$ & $\max$ & defauht & unit & description of component \\
\hline \hline rframe & name & & & ' & & $\begin{array}{l}\text { name of reference frame } \\
\text { on the same part }\end{array}$ \\
\hline origin & param (3) & & & $3 * 0$. & {$[\mathrm{m}]$} & origin of frame \\
\hline axleseq & int (3) & -3 & 3 & $1,2,3$ & & $\begin{array}{l}\text { axlesequence of } \\
\text { elementary rotations }\end{array}$ \\
\hline angles & param (3) & $-2 \pi$ & $2 \pi$ & $3 * 0$. & {$[\mathrm{rad}]$} & rotation angles \\
\hline
\end{tabular}

Fig. 4. Objects of the class "frame" in RSYST representation

\section{THE MULTIBODY SYSTEMS DATAMODEL}

The method of multibody systems is based on a finite set of elements like bodies and foints shown in Ref. [13] or by Roberson and Schwertassek [14). These elements can be used to define a unique datamodel applying the classes and objects of a software engineering tool like RSYST.

The datamodel has been defined as a standardized basis for all kinds of computer codes by Otter, Hocke, Daberkow and Leister [15]. The following assumptions were agreed upon:

1. A multibody system consists of rigid bodies and ideal joints. A body may degenerate to a particle or to a body without inertia. The ideal joints include the rigid joint, the joint with completely given motion (rheonomic constraint) and the vanishing joint (free motion).

2. The topology of the multibody system is arbitrary. Chains, trees and closed loops are admitted.

3. Joints and actuators are summarized in open libraries.

4. Subsystems may be added to existing components of the multibody system.

A datamodel for elastic bodies is under development and will be completely compatible with the rigid body datamodel.

A multibody system as defined is characterized by the class mbs and consists of an arbitrary number of the objects of the classes part and interact, see Fig. 5.

The class part describes rigid bodies. Each part is characterized by at least one body-fixed frame, it may have a mass, a center of mass and a tensor of inertia.

The class interact describes the interaction between a frame on part $a$ and a frame on part b. The interaction may be realized by a joint, by 


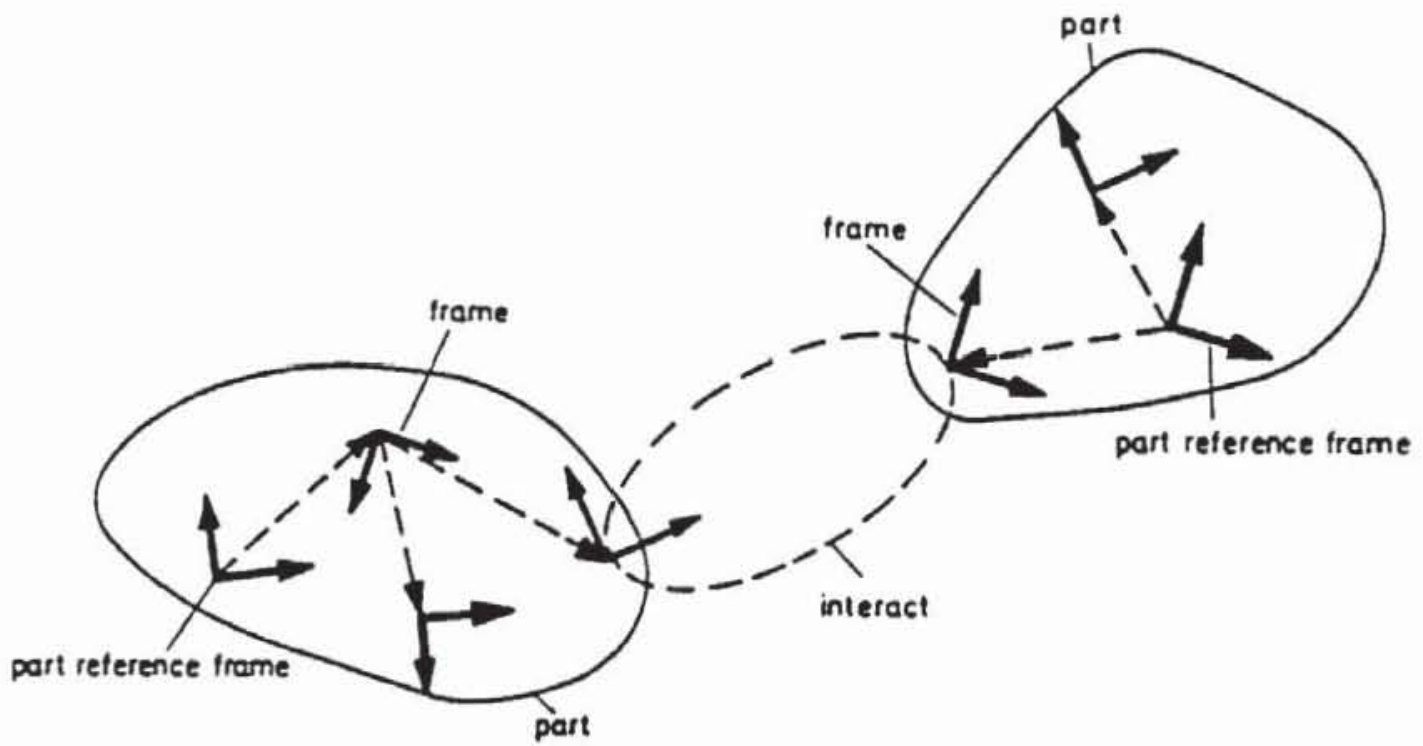

Fig. 5. Definition of a multibody system

an force actuator or a sensor resulting in the clasees joint, force or sensor, respectively. Thus, the class interact is characterized by two types of information: the frames to be connected and the connecting element itself, gee Fig. 6 .

According to the assumptions, the datamodel represents a holonomic, rheonomic multibody system. If the formalism selected uses a minimal number of coordinates, Like NEWEUL, then, the resulting equations of motion read as

$H(y, t) \ddot{y}+k(y, y, t)=q(y, \dot{y}, t)$.

Here, $y$ is the position vector and $t$ the time, $M$ means the generalized inertia matrix, $k$ and $q$ represent the generalized gyroscopic and applied forces. However, Eq.(1) which represents a set of pure differential equations is not a consequence of the datamodel. By an appropriate formalism from the same datamodel a set of differential-algebraical equations may be obtained, too.

In addition to the mechanical elements of a multibody system, there exists also the possibilty to use the general motion of dynamical systems, e.g.,

$\dot{x}=f(x, u, t, p)$

$z=g(x, u, t, p)$

where $x$ is the state vector, $z$ the output vector, $u$ the input vector, $t$ the time and $p$ the parameter vector. Further, $f$ and $g$ mean nonlinear vector functions. For more details see Ref. [15]. 


\begin{tabular}{|l|l|l|}
\hline class: & \multicolumn{3}{|c|}{ interact } \\
\hline name & class & description of component \\
\hline \hline connect & connect & frames to be connected \\
\hline member & member & connecting element \\
\hline
\end{tabular}

\begin{tabular}{|l|l|l|l|l|l|l|}
\hline class: & connect \\
\hline name & type & min & max & default & unt & description of component \\
\hline \hline apart & name & & & & & name of apart \\
\hline aframe & name & & & ' & & name of aframe on apart \\
\hline bpart & name & & & & & name of bpart \\
\hline bframe & name & & & ' & & name of bframe on bpart \\
\hline
\end{tabular}

\begin{tabular}{|l|l|l|}
\hline class: & \multicolumn{2}{|l|}{ member } \\
\hline name & class & description of component \\
\hline \hline joint & joint & joint element on interact object \\
\hline force & setof (force) & force elements on interact object \\
\hline sensor & setof (sensor) & sensor elements on interact object \\
\hline
\end{tabular}

Fig. 6. The class interact

\section{THE ILTIS BENCHMARK PROBLEM}

The standardized object oriented datamodel for multibody systems as described in the previous gection is very well qualified for vehicle dynamics applications. In particular, it offers the possibility for a very convenient benchmark analysis for all programmes adjusted to the modular software system under development of the auspices of the German Research Council which mean public domain software.

A first example is a van treated in detail by otter, Hocke, Daberkow and Leister [15], see Fig. 7. The van consigts of four bodies easily identified from the RSYST database.

A second example is the Iltis vehicle chosen by IAVSD as benchmark and more precisely described by Kovanda [16]. Moreover, the second IAVSD benchmark, the five point wheel suspension, can be represented by the RSYST database, too. Then, it is very gimple to analyse the Iltis vehicle with a more sophisticated suspension. Fig. 8 shows the Iltis vehicle and the corresponding database structure while in Fig. 9 the five point wheel suspension is presented. 


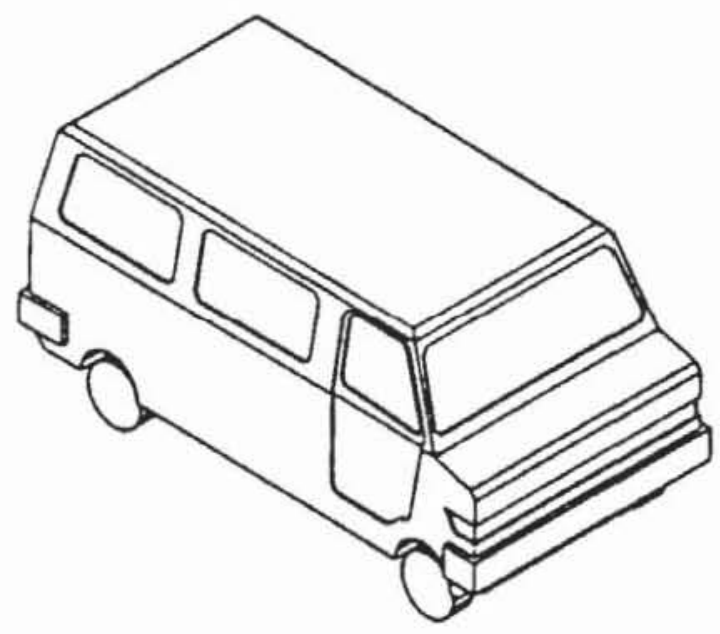

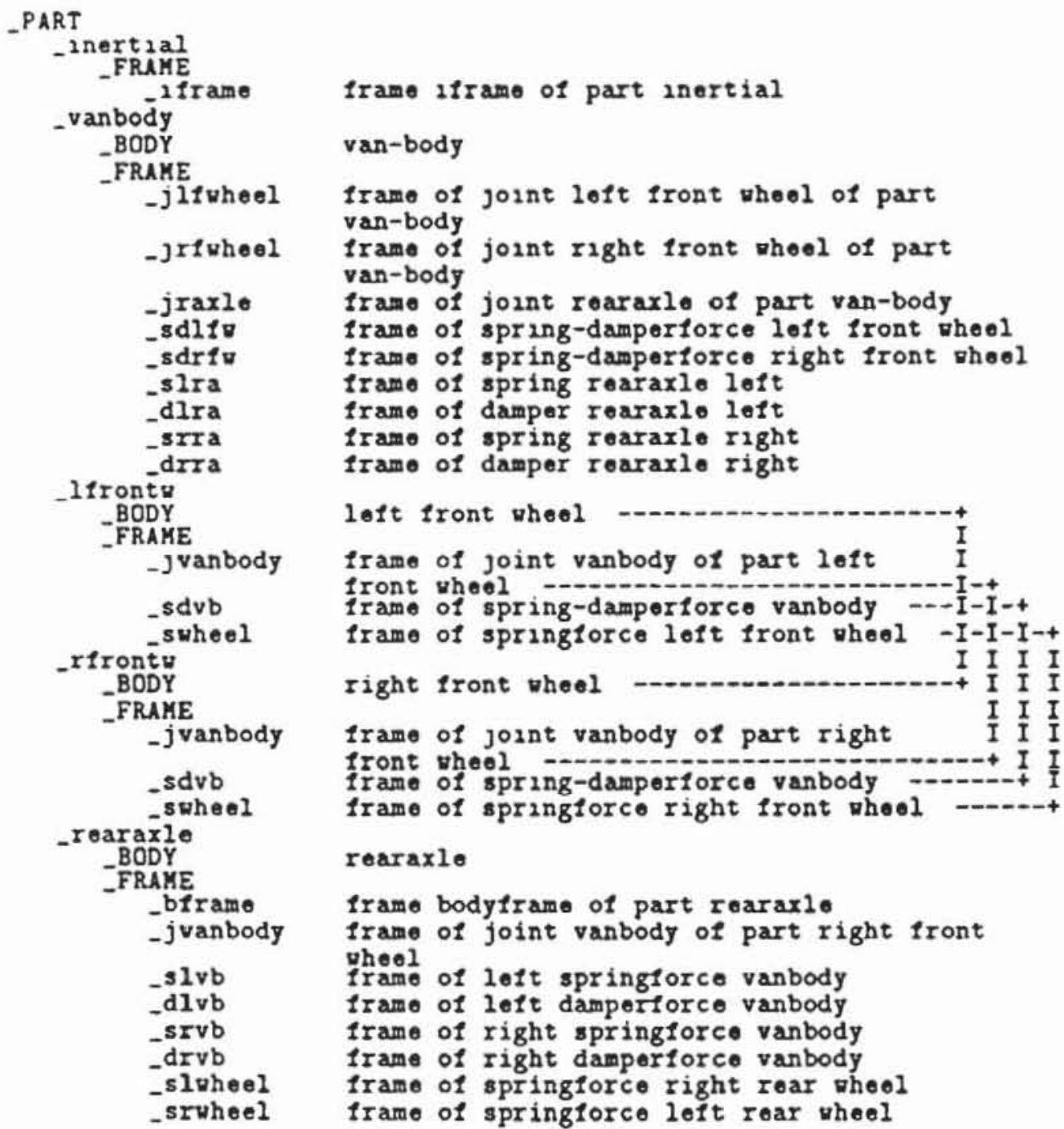

Fig. 7. Drawing and database atructure of a van 

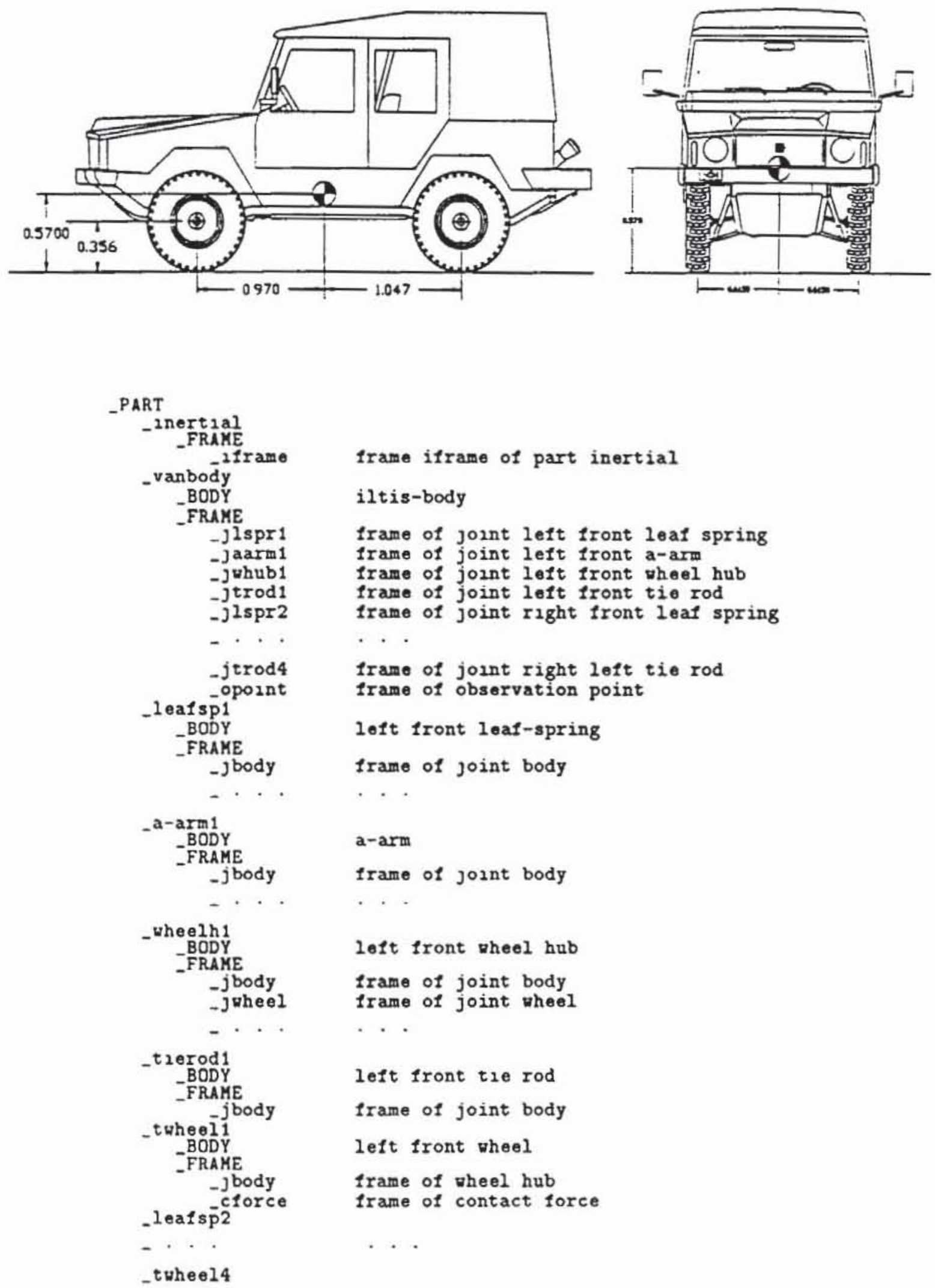

Fig. 8. Iltis vehicle and corresponding database structure 

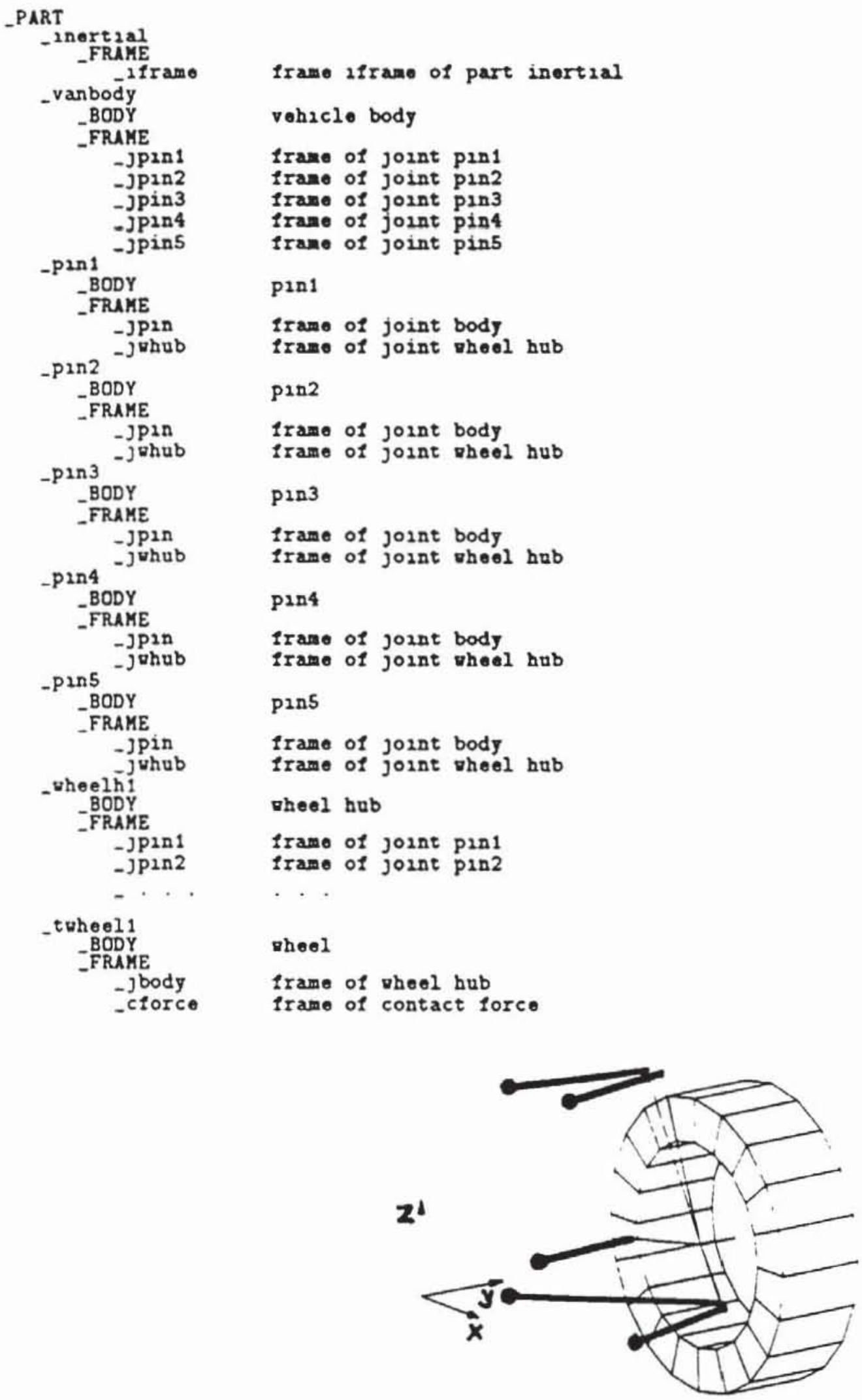

Fig. 9. Wheel suspension and corresponding database structure 


\section{CONCLUSION}

The modular software engineering design and the related database concept of the German multibody systems project of fers for the first time the prospect reliable benchmarking of vehicle dynamics simulations. Even if the RSYST database is not used, the unique and clear definition of all input and parameter data seems to be a great help with respect to finding standarda for vehicle dynamics simulations.

It has to be mentioned that at the time being within the German project special modules for the wheel/rail and tire/road contact are not to be developed. However, the modular concept is well qualified to add programes dealing with vehicle wheels and tires. The final results of the German Project will be presented early in 1993 on an international meeting.

\section{REFERENCES}

1. Magnus, X. (ed.), Dynamics of Multibody Systems. Berlin/..., Springer-Verlag 1978.

2. Slibar, A. and Springer, H. (eds.), Dynamics of Vehicles on Roads and Railway Tracks, Swets and zeitlinger 1978.

3. Haug, E. J. (ed.), Computer Aided Analysis and Optimization of Mechanical System Dynamics. Berlin/..., Springer-Verlag 1984.

4. Kortüm, w. and Schiehlen, w., General Purpose Vehicle System Dynamics Software Based on Multibody Formalisms. Vehicle System Dynamics 14(1985), p. 229-263

5. Bianchi, G. and Schiehlen, w. (eds,), Dynamics of Multibody Systems. Berlin/..., Springer-Verlag 1986.

6. Kortüm, w. and Sharp, R. S., A Report on the State-of-Affairs on "Application of Multibody Computer Codes to Vehicle system Dynamics". Vehicle System Dynamics 20(1991), p. 177-184.

7. Schiehlen, w. (ed.), Multibody Systems Handbook. Berlin/..., Springer-Verlag 1990.

8. Kortüm, W., de Pater, A. D. and Sharp, R. S., Application of Multibody Computer Codes to Vehicle System Dynamics. Vehicle System Dynamics, to appear.

9. Meinke, P. (ed.), DFG-Schwerpunktprogramm "Dynamik von Mehrkörpersystemen". 3. Berichtskolloquium (24./25. Januar 1991, Augsburg). München: MAN Technologie AG 1991.

10. Lang, U., Erstellen von Anwendungsmoduln in RSYST. Stuttgart, Rechenzentrum der Universität Stuttgart 1988.

11. Loebich, I., Einführung in RSYST. Stuttgart, Rechenzentrum der Universität Stuttgart 1988 .

12. Rühle, R. et al., RSYST Unterprogramm- und Modul-Dokumentation, Version 3.5.0. Stuttgart, Rechenzentrum der Universität 1988.

13. Schiehlen, W., Technische Dynamik. Stuttgart, Teubner 1986.

14. Roberson, R. E. and Schwertassek, R., Dynamics of Multibody Systems. Berlin/..., Springer-Verlag 1988.

15. Otter, M., Hocke, M., Daberkow, A. und Leister, G., Ein objektorientiertes Datenmodell zur Beschreibung von Mehrkörpersystemen unter Verwendung von RSYST. Institutsbericht IB16. Stuttgart, Institut B für Mechanik 1990. 
16. Kovanda, J., Modelling and Simulation of the Iltis Vehicle with NEWEUL/NEWSIM. ZWischenbericht zB-58. Stuttgart, Institut $B$ für Mechanik 1990. 